\title{
Research on Driver's Speed Control Behavior at Urban Signalized Intersection
}

\author{
Dong Wang \\ School of Vehicle Engineering, Xi’an Aeronautical University \& Key Laboratory for Automotive \\ Transportation Safety Enhancement Technology of the Ministry of Communication, Xi'an, 710000
}

Keywords: Driver Speed Control Behavior, Urban Signalized Intersection

\begin{abstract}
The non-mixed characteristics of the intersection directly lead to it become the bottleneck of road traffic, becoming a high-speed road accident. When crossing the intersection of the highway, the driver must be able to screen the key information and control the vehicle speed rationally to effectively avoid the conflicts of different directions. Therefore, studying the speed control mode and visual search mode of the driver at the plane intersection is of great importance to guide the driver safely through the intersection, effectively avoid the traffic conflict, reduce the traffic accident and improve the traffic capacity of the entire road network. Based on the urban flat signalized intersection, this paper makes a real vehicle test in a real traffic environment, records the driving speed of the driver approaching the intersection and crossing the intersection, and uses the eye tracker to record the driver's visual search Behavioral, quantitative analysis of driver behavior at the intersection of driving behavior. The statistical analysis of the test data will be a complete intersection of the driving process in accordance with the parking line is divided into approaching the intersection and through the intersection of two parts, and according to different ways of crossing the intersection of straight line, turn left and right Then, classify each category according to the driving status before intersection, then classify them into waiting, queuing and waiting in line. According to different situations, analysis of driver approaching the intersection and through the intersection of speed control mode and visual search mode.
\end{abstract}

\section{Introduction}

The increasingly serious traffic environment and the rapid growth of car ownership will inevitably bring opportunities to traffic accidents. Environment, people, cars, roads, lack of cooperation is likely to lead to the occurrence of traffic accidents. In the entire road network, although only a small part of the intersection, but it is prone to traffic accidents. Accidents in the United States accounted for the total number of accidents at the intersection of the former Federal Republic of Germany at the intersection and those at the urban intersections accounting for the data on fatal accidents in Japan in the past year occurred at and near the intersection and at the plane intersection in our country Accidents account for around the city's traffic accidents. The reason why intersections are called accident-prone places is that the basic reason for this is that they converge the traffic streams here. The traffic participants at the intersections mainly include pedestrians and bicycles, electric bicyclists, etc. besides the drivers themselves. Pedestrian crossing is often characterized by uncertainty. Bike and electric vehicles, because of their fast start-up speed, instability and expansibility at low speed, also affect the traffic safety of intersections at all times. If the driver can not fully grasp the traffic information and then control the vehicle speed to avoid, it is most likely to lead to traffic accidents. However, pilot speed control and informational reserves approaching the intersection greatly affect safety through the intersection. For example, if the driver can not observe the traffic signal in time, failure to take braking in time should probably trigger a red light. This will undoubtedly cause great traffic conflicts at the intersection and affect the traffic order. This article is based on the urban flat signal intersection, through the real traffic test in real traffic environment, quantitative analysis of the driver approaching the intersection and through the intersection of the speed control mode and visual search mode, through the driver speed control Way and visual search mode of research, you can roughly grasp the driving intersection of the unsafe factors. If the pilot can be effectively instructed and the safety hazard relieved as soon as 
possible, it will be possible to take preventive measures to improve driving safety, greatly reduce traffic accidents, reduce the loss of life and property, and promote law and order stability and social harmony.

\section{Intersection features}

The definition of traffic conflict first came from the first annual conference on international traffic conflicts in Norway. It means that road users of relative movement are close to each other in time and space. If they are allowed to develop naturally, they may collide and turn this The phenomenon is called traffic conflict. Driver driving vehicles passing through the intersection, due to the interaction with other participants, will inevitably produce diversion and confluence. Traffic conflicts can occur if a certain point at the intersection brings together traffic flows of different flows at the same time. Traffic conflicts can usually be classified by severity, object of conflict and direction of movement, respectively.

The non-mixed characteristics of the intersection directly lead to it become the bottleneck of road traffic, to create favorable conditions for the occurrence of Jiayoukou accident. Coupled with the driver's poor driving behavior, the number of accidents increased significantly. Traffic accidents caused by driving errors up to one, only speeding can lead to the total number of traffic accidents. Etc. Through the model prediction, it is concluded that the drivers 'cognition errors or pedestrians' interferences at the intersections will most likely lead to vehicle rear-end or scraping, while the vehicle rear-end caused by the interference caused by driving while on the phone significantly increases. The so-called unsafe driving behavior, refers to the driver to subjectively achieve their own goals not conducive to safe driving behavior, such as running a red light, illegal, speeding and so on. The picture below shows the bad car driving behavior released by the Ministry of Public Security according to the severity of the accident, which is derived from the statistical analysis of the national traffic accidents.

Degree should be lower than the claw, if the intersection of narrow pavement, blind area, try to control the vehicle speed in one. Vehicles turning at the intersection should turn on the turn signals outside the stop line, select the lane according to the road guide arrow, do not hit the steering wheel to change the lane, and should not be the first to start in the stop, but not suddenly take the acceleration through the intersection. Vehicles waiting to pass through the intersection do not turn off the engine and ensure that the traffic lights start in time. Intersections often lead to poor visibility due to surrounding building facilities, etc. At this moment, the driver needs to obtain visual information through the corner reflector in time. However, the corner reflector is smaller than the actual situation because of the dead corner and is reversely mapped The driver can only take the traffic information obtained through the curve mirror as a reference, and must take a certain amount of thinking and response to correctly grasp the dynamic traffic conditions. The main reason lies in reducing the speed in advance so as to be able to cope with various kinds of sudden conflicts.

The non-mixing feature of the intersection makes it become a gathering place for traffic conflicts. Drivers who want to drive safely and smoothly must observe the dynamic traffic conditions at any time, and control the speed rationally.

\section{Study on Driver Behavior Characteristics When Approaching Intersection}

According to the above description, the straight-ahead approach intersection includes the straight-ahead pass, the straight-line stoppage, and the waiting line waiting in the line. Starting from this situation, the research on driver driving behavior of straight-ahead intersection starts from this situation and carries out in-depth analysis. The driver ran at a high speed when it was far from the intersection, and quickly reduced the vehicle speed as the distance was reduced from the intersection. At the intersection, the driver maintained a steady low-speed ride. The driver remained at a high speed even beyond the speed limit at a distance away from the intersection, slightly slowed down at the distance intersection and accelerated near the intersection. The reason why the driver accelerated through video recording was probably due to the low traffic flow and the front 
Unobstructed view and interference. The driver ran at a steady speed outside the intersection, followed by a continuous deceleration, about to reach the intersection at low speed. The driver approached the intersection with a large fluctuation in speed. The driver has an acceleration behavior at a distance away from the intersection. After a period of steady running, the driver begins to decelerate at the distance intersection and reaches the intersection about a low speed of the knife. By analyzing the means and standard deviations of the average speed and the standard deviation of drivers approaching the intersection, it is found that the difference of the driver's speed from the intersection is the smallest, that is, the driver's choice of speed is the same, while the driver's speed difference is the largest . This is driven by the driver at a distance intersection, some drivers take the deceleration, while some drivers to accelerate. In the process of approaching the intersection from the intersection, all of the above drivers have an over speed limit. Individual drivers did not slow down properly until they reached the intersection, and even chose to accelerate as they approached the stop line. Both of these actions pose a certain threat to safety through the intersection. In order to ensure safety, timely advice and guidance should be given to such drivers.

Turn right and cross the intersection again and again. Pass the driver before the intersection, without stopping, and turn right from the stop line through the intersection. The curve shows how the speed varies with the driving distance as the driver turns right from the parking lane into the intersection and passes through the intersection. As can be seen from the figure, right turn right through the intersection of the case of the right turn are basically the first deceleration after the speed of entry into the intersection of a large difference in speed, out of the intersection of the speed difference is not big, the middle of the deceleration point position is different. Due to the different traffic conditions at the intersections, the pedestrians and vehicles have different degrees of interference. In addition, the driver's driving conditions before the intersection are different, and the driver's speed at entering the intersections is quite different. The description of the previous paragraph awaiting the right turn through an intersection stops waiting for the driver to stop at the intersection parking line and starts the vehicle to turn right through the intersection when the green light is released. The curve represents the change of the speed with the distance traveled by the driver from the stop line to start the vehicle entering the intersection to the right turning out of the intersection. As can be seen from the figure, there is a clear law of turning right at the driver's intersection when waiting for a right turnaround. The speed fluctuation clearly shows a process of accelerating from the parking line to the intersection, then driving steadily and accelerating Exit the intersection. The average acceleration is resistance, compared with the driver's acceleration of entering the intersection of Jiaokou and Koukou, the exit of the intersection is gentle, and the relative acceleration of each driver is relatively different. It is known from the foregoing that turning right and waiting in line through the intersection means that the driver stops waiting at a distance from the intersection and starts turning right through the intersection when the green light is released. The process is divided into two sections. The section from the stop state to the parking lane at the intersection belongs to the case of waiting for a right turn before the intersection, and the latter section is from the parking lane to the intersection to the right mouth. The curve shows the change in speed with the distance traveled in the later period. As can be seen from the figure, there is a clear law of turning right at the driver's intersection waiting in the right direction of waiting in line, slowly decelerating into the intersection, then accelerating out of the intersection.

\section{Conclusion}

This paper attempts to find the law of driving at the driver's intersection, which requires the support of a large sample. Due to the limitation of time and objective reason, this condition is not satisfied. In the future to carry out more in-depth study, may be appropriate to increase the sample. In this paper, the research on driver behavior at intersection is in the exploration stage, and no consideration has been given to the factors affecting the driving behavior such as traffic volume, driver's age difference and driving experience difference. In order to ensure the objectivity and persuasion of laws, we should try our best to summarize the law thoroughly. 


\section{Acknowledgements}

Fund Project: Central University of basic scientific research operating expenses special funds (310822171116)

\section{References}

[1] Meng Yalan, Pan Fuquan. Research Status and Trend of Traffic Illegal Monitoring to Drivers [J] .Shandong Transportation Science and Technology. 2016 (02)

[2] Pang Fuquan, Zhang Lixia. Driver Behavior Modeling At Countdown Signal Intersections Considering Vehicle Value [J]. Transportation Systems Engineering and Information. 2016 (02)

[3] Pan Fuquan. The Modeling and Analysis of Traffic Track Confirmation of a County Tunnel [J] .Journal Of China Safety Science 2015. (07)

[4] Liu Tao. A Review of The Applications o Intelligent Traffic At Signalized Junction [J] .Journal of Qingdao University of Science And Technology. 2015 (03)

[5] Pei Jian. Electronic Police System in Intelligent Transportation [J]. China Security. 2014 (06)

[6] Wei Liying, Wang Zhilong, Wu Ronghua. Study and Modeling of Lane-Changing Behavior at Intersection [J]. Acta Physica Sinica. 2014 (04) 